Methods: Ten HCCs, which were diagnosed by biopsy, treated by TACE and consequently resected and 36 HCCs without TACE, biopsy and resection matched were selected from hepatic resections between 2001 and 2014 at Severance Hospital. Total necrotic HCCs after TACE were excluded. Immunohistochemistry for stemness markers (K19, EpCAM and CD133) and hypoxic marker (CA-IX) were performed.

Results: The expression rate of K19, EpCAM, CD133 and CAIX were well matched between biopsied and resected HCCs with specificity ranging from 82 to $100 \%$. K19 expression was well correlated with that of CA-IX. Biopsied HCCs, done before any treatment showed no difference in the incidence or extent of expression of stemness markers and CA-IX between TACE and non-TACE groups. TACE treated/resected HCCs showed higher incidence of EpCAM expression compared to those without ( $p=0.033$ ), whereas the incidence of K19, CD133 and CA-IX expression showed no significant difference between two groups. The extent of EpCAM and K19 expressing tumor cells increased in TACE treated/resected HCCs compared to those without ( $p=0.028$ and $p=0.049$ ). K19 and CAIX positive patients on biopsied HCCs of both groups showed lower survival rate ( $p=0.013$ and $p=0.008$, respectively).

Conclusions: It is considered that K19 expression is related to CAIX, and hypoxic microenvironment induced by TACE increases the extent of K19 expressing tumor cells rather than converting K19-negative HCC to K19-positive HCC. Therefore, checking K19 and CA-IX expression on HCC biopsy before TACE is suggested to be good markers predicting HCC outcome.

\section{CLINICOPATHOLOGIC AND PROGNOSTIC SIGNIFICANCES OF miRNAlet-7 $\alpha$ EXPRESSION AND ITS ASSOCIATION WITH AR STATUS IN BREAST CARCINOMAS}

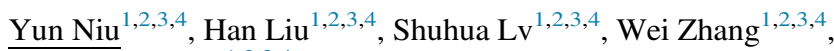
Tongxian Zhang ${ }^{1,2,3,4}$

${ }^{1}$ Key Laboratory of Breast Cancer Prevention and Therapy, Ministry of Education, ${ }^{2}$ Key Laboratory of Cancer Prevention and Therapy, Tianjin, ${ }^{3}$ Cancer Hospital, Tianjin Medical University, and ${ }^{4}$ National Clinical Research Center for Cancer, China

Background: MiRNAlet-7a as a member of the let-7 family, has been widely considered as a tumor suppressor because the let-7a is down-regulated in many carcinoma progression. Androgen receptor (AR) was expressed in breast cancer as a significant prognostic factor (including our data).

Aims: To analyze the associations of miRNAlet-7a expression with the clinicopathologic characteristics and prognosis in different AR status.

Methods: One hundred sixty eight cases with invasive ductal carcinoma were randomly selected, the miRNAlet-7a expression was detected by in-situ hybridization, and the expression of AR, ER, and Ki67 were detected by immunohistoehemistry. These cases were divided into AR+ and AR-groups, and the results were analyzed by statistics.

Results: The positive rate of miRNAlet-7a expression was $70.2 \%$. There were significant differences in the deferent grad and different AR status $(p=0.002,0.000)$. But, there was no significant difference in age, menstrual status, size, pTNM, node metastasis, ER, p53, ki67, HER2 (all $p>0.05$ ). Let-7a expression correlate positive with $\mathrm{AR}(p=0.000)$. In AR+ group, there was no significance found between the expression of miRNAlet-7a and clinicopathologic characteristics, in AR - group conversely, miRNAlet-7a was associated with histological grade $(p=0.004)$ The let-7a expression was all associated with better disease-free survival $(p=0.000)$ in the two groups, respectively. The multivariate-analysis showed it was a significant predictor of diseasefree survival $(p=0.000)$.

Conclusions: miRNAlet-7a may play important roles in the breast carcinomas of different AR status. It's as indicator of predicting prognosis and maybe a new therapeutic target.

\section{PDL1 EXPRESSION IN TRIPLE-NEGATIVE BREAST CANCER IS ASSOCIATED WITH TUMOUR- INFILTRATING LYMPHOCYTES AND IMPROVED OUTCOME}

Rhiannon K. Beckers ${ }^{1,2}$, Christina Selinger ${ }^{1}$, Ricardo Vilain ${ }^{1,4,5}$, Jason Madore $^{5}$, James S. Wilmott ${ }^{4,5}$, Kate Harvey ${ }^{1,2}$,

Anne Holliday $^{2}$, Caroline L. Cooper ${ }^{1,4}$, Elizabeth Robbins ${ }^{1}$, David Gillet $^{6}$, Catherine W. Kennedy ${ }^{6}$, Laurence Gluch ${ }^{4,6,8}$, Hugh Carmalt ${ }^{4,6,7}$, Cindy Mak ${ }^{7}$, Sanjay Warrier ${ }^{3,4,7,9}$, Harriet E. Gee ${ }^{10,11}$, Charles Chan ${ }^{4,13}$, Anna McLean ${ }^{4}$, Emily Walker ${ }^{4}$, Catriona M. McNeil ${ }^{4,12}$, Jane M. Beith ${ }^{4,12}$, Alexander Swarbrick ${ }^{2,3}$, Richard A. Scolyer ${ }^{1,4,5}$,

Sandra A. O'Toole ${ }^{1,2,3,4}$

${ }^{1}$ Department of Tissue Pathology and Diagnostic Oncology, Royal Prince Alfred Hospital, Camperdown, ${ }^{2}$ The Kinghorn Cancer Centre and Cancer Research Program, Garvan Institute of Medical Research, ${ }^{3}$ St Vincent's Clinical School, Faculty of Medicine, University of New South Wales, Darlinghurst,

${ }^{4}$ Sydney Medical School, University of Sydney, ${ }^{5}$ Melanoma Institute Australia, Sydney, ${ }^{6}$ The Strathfield Breast Centre, Strathfield, ${ }^{7}$ Department of Breast Surgery, Royal Prince Alfred Hospital, Camperdown, ${ }^{8}$ Department of Breast and Endocrine Surgery, Concord Repatriation General Hospital, Sydney, ${ }^{9}$ Department of Surgery, Prince of Wales Hospital, Randwick, ${ }^{10}$ Department of Radiation Oncology, Sydney Cancer Centre, Royal Prince Alfred Hospital, Camperdown, NSW, Australia; ${ }^{11}$ Department of Oncology, Weatherall Institute of Molecular Medicine, University of Oxford, John Radcliffe Hospital, Oxford, United Kingdom; ${ }^{12}$ Department of Medical Oncology, Chris O'Brien Lifehouse, Camperdown, and ${ }^{13}$ Anatomical Pathology Department, Concord Repatriation General Hospital, Concord, NSW, Australia

Purpose: Triple-negative breast cancer lacks expression of estrogen receptor, progesterone receptor and human epidermal growth factor receptor and patients generally have a poor outcome; there is a pressing need to identify more effective therapeutic strategies. Clinical trials targeting PD1/PDL1 in melanoma and non-small cell lung cancer have reported high response rates, and tumoural PDL1 expression has been suggested as a potential biomarker to enrich for patient response to these treatments. There is only very limited data to date reporting the expression of PDL1 in triple-negative breast cancer. This study characterises PDL1 expression patterns and tumour-infiltrating lymphocytes in primary triple-negative breast cancer. Methods: PDL1 immunohistochemistry was performed on 163 primary triple-negative breast cancers and assessed in the tumour (membranous and cytoplasmic compartments) as well as immune cells in the stromal compartment. Tumour-infiltrating 